Cambouis, la revue des sciences sociales aux mains sales

\title{
Négocier sa place auprès des sans-abri
}

\section{L'exemple d'une immersion} ethnographique dans le monde de la rue

\section{Thibaut Besozzi}

Université de Lorraine, 2L2S ; Université de Reims, CEREP

Thibaut.besozzi@univ-lorraine.fr

Thibaut.besozzi@univ-lorraine.fr

Résumé:En partant d'une étude menée en immersion ethnographique pendant 8 mois dans le monde de la rue, auprès des sans-domiciles présents à Nancy, cet article développe les procédés de négociation qui ont été déployés pour entrer sur le terrain, s'y maintenir et y obtenir, tant bien que mal, les données nécessaires à la réalisation de l'enquête. La diversité des personnes en situation de rue oblige le chercheur à ajuster sa présentation de soi pour développer des relations personnalisées avec ses enquêtés et accéder aux coulisses du monde de la rue. L'article distingue la phase stratégique d'anticipation du terrain et la phase tactique d'adaptation au milieu, en situation, destinée à maximiser les possibilités d'entrer dans des relations de réciprocité affinitaires. En filigrane, émerge une interrogation constante de la relation d'enquête, entre la proximité relationnelle et l'asymétrie structurelle du rapport enquêteur/enquêté.

Date de publication : 30/06/2021

Ethnographie, négociation, relation d'enquête, proximité relationnelle, distance sociale

Dossier: Pratiques et politiques de la négociation pour accéder et se maintenir sur un terrain d'enquête

Comment citer : $10.52983 /$ crev.vi0.73

Licence: Cambouis publie ses contenus selon les termes de la Licence Creative Commons Attribution - Pas d'Utilisation Commerciale - Pas de Modification 4.0 International. Les auteurices gardent leurs droits de propriété intellectuelle pleine et entière sur leurs articles. 


\section{Négocier sa place auprès des sans-abri}

\section{L'exemple d'une immersion ethnographique dans le monde de la rue}

\section{Thibaut Besozzi}

Université de Lorraine, 2L2S ; Université de Reims, CEREP

Thibaut.besozzi@univ-lorraine.fr

Cet article entend présenter la manière dont j'ai négocié ma place sur le terrain lors d'une enquête par immersion ethnographique menée à Nancy, ville centre d'une agglomération de 300000 habitants localisée dans le nord-est de la France. « Négocier sa place » consiste à déployer un ensemble de techniques et d'attitudes pour entrer sur le terrain, s'y maintenir et accéder aux moments et situations où se jouent des activités, des relations et des enjeux que seule l'ethnographie est à même d'appréhender. La «négociation » présentée ici ne se résume pas à une argumentation orale destinée à trouver un accord entre deux parties mais relève plutôt de la mise en scène de soi pour gagner la confiance des enquêtés. En tant que tel, mon propos ne se contente pas d'illustrer des outils méthodologiques relatifs à l'ethnographie mais se donne également pour objectif de défendre la fécondité de l'approche ethnographique, qui plus est lorsqu'elle est pratiquée en immersion (Leroux, Neveu, 2017).

Une première expérience de recherche ethnographique m'a permis d'être approché par les associations responsables de l'action sociale d'urgence et de réinsertion à Nancy afin d'effectuer une recherche qualitative sur les SDF et les sans-abri ${ }^{1}$. La méthode d'immersion ethnographique avait retenu leur attention tant elle permet de produire un diagnostic sociologique apportant une connaissance « de l'intérieur » sur les publics dits « en errance », leur vie quotidienne et leur organisation collective. Financée par la Direction Départementale de la Cohésion Sociale (DDCS), l'association Accueil et Réinsertion Sociale (ARS), la Métropole du Grand Nancy et le Centre Communal d'Action Sociale (CCAS) de Nancy, la recherche s'est étendue sur 20 mois (de septembre 2017 à avril 2019) et se donnait pour objectif de dégager des préconisations opérationnelles pour améliorer l'offre d'accueil et d'accompagnement des personnes chroniquement ou durablement à la rue. L'adaptation des modalités d'intervention sociale passe ici par une fine connaissance des publics visés par les dispositifs d'urgence sociale et de réinsertion ; une connaissance localisée et située que permettent précisément l'ethnographie et l'approche pragmatique (Joseph, 2007). Il semble que dans le domaine de la recherche-action, cette perspective ait beaucoup à apporter si elle est directement indexée aux objectifs et volontés d'action locale de la part des commanditaires. Sur le plan scientifique, il convient néanmoins de reconnaître le localisme intrinsèque de la méthode ethnographique qui ne peut rendre compte que de réalités situées - dans le temps et dans l'espace et invite donc à la précaution dans l'entreprise de généralisation des résultats.

Pour être plus précis, il s'agissait d'analyser le « monde de la rue » à partir d'un engagement actif dans la vie quotidienne des personnes sans-domicile ou, autrement dit, d'une observation participante au long cours. En effet, si les travailleurs sociaux ont une connaissance assez poussée de leurs publics dans la mesure où ils les côtoient et les accompagnent (parfois depuis plusieurs années), cette connaissance reste limitée aux cadres institutionnels et
1 Du fait qu'elle s'intéresse prioritairement aux publics et dispositifs d'urgence sociale, ma recherche s'est plutôt focalisée sur des situations de sans-abrisme (Choppin, Gardella, 2013) que sur des personnes «SDF lato sensu » (Damon, 2012). L'enquête inclut également les individus qui se disent « Zonards » ou « Routards » et se situent à la charnière entre le sans-abrisme et la revendication active d'un mode de vie alternatif et marginalisé (voir le ${ }^{\circ} 171$ de la revue Espaces et sociétés, paru en 2017, ainsi que l'ouvrage de T. Pimor, 2014). 
interactionnels qui structurent la relation et la perception mutuelle des uns et des autres. C'est pourquoi il importait d'atteindre une autre connaissance de ces personnes, touchant à tout ce qui se déroule en dehors des services sociaux ou, autrement dit, à « l'envers du décor » ou au « texte caché » (Scott, 2008). Il s'agissait donc pour moi de reconstituer le réseau d'interconnaissance des personnes attenantes au monde de la rue, d'en clarifier la complexité à partir d'une typologie des diverses personnes rencontrées, de dégager les structures morales, spatio-temporelles et relationnelles qui consolident ce monde social, mais aussi de rendre compte des pratiques quotidiennes et des enjeux identitaires qui traversent la survie des sans-abri (Besozzi, 2019). C'est ainsi que se donne à voir un monde possédant sa cohérence interne, ses formes d'organisation informelle, ses logiques de rationalité propres à la survie, le tout opérant à travers les actions quotidiennes d'individus pris dans une tension entre perdre la face et sauver la face (Goffman, 1973a ; 1973b ; 1974); une tension à laquelle ils sont inévitablement confrontés dans le cadre du « maintien de soi » (Pichon, 2010).

Accéder à cet ensemble de dimensions nécessitait l'immersion ethnographique afin d'être aux premières loges pour rendre compte de la vie quotidienne, collective et individuelle des sans-abri qui fréquentent $\mathrm{Nancy}^{2}$. Pour ce faire, il fallait d'abord négocier pour pouvoir m'intégrer et me maintenir sur ce terrain qui ne pouvait être seulement survolé par entretiens compte tenu des objectifs de la recherche.

Plus concrètement, je me suis immergé dans la vie quotidienne du monde de la rue pendant presque 8 mois, de septembre 2017 à avril 2018, tout en continuant d'être en contact avec une dizaine d'informateurs après cette période (et aujourd'hui encore). À la fin de cette phase intensive de terrain, j'avais passé plus de 300 heures auprès des sans-abri rencontrés, répertorié presque 200 personnes dans un tableau compilant les informations collectées sur chacune (dont une centaine de cas sont assez complètement renseignés), j'avais suivi de près une cinquantaine de personnes et interrogé longuement (avec ou sans enregistrement) une trentaine d'entre elles. Des liens de familiarité, parfois d'amitié, se sont développés entre eux et moi, non sans conflits par moments, tandis que j'ai visité une petite dizaine de squats plus ou moins éphémères, plusieurs installations temporaires dans des parkings souterrains mais aussi des logements et la quasi-totalité des services d'urgence sociale (caritatifs ou institutionnels) présents sur le territoire de la ville (qu'il s'agisse de services alimentaires, d'accueils de jour, d'hébergements d'urgence ou de centres de réduction des risques liés à la toxicomanie). Comme cela se fait de manière traditionnelle et indispensable en ethnographie, je remplissais quasi quotidiennement un journal de bord où se côtoient pêle-mêle, sur plusieurs centaines de pages, des descriptions de scènes vécues, des ressentis personnels, des interprétations à chaud, des fiches individuelles ainsi que des schémas et tableaux divers. Enfin, j'ai pu bénéficier de la collaboration des travailleurs sociaux pour obtenir des informations individuelles compilées dans les dossiers d'accompagnement et dans les archives statistiques répertoriant les usagers de trois services d'urgence que sont l'accueil de jour, le service alimentaire du soir et l'hébergement de mise à l'abri accueillant principalement les « appelants 115 ».

Voyons comment s'est déroulée cette immersion et quelles ont été les techniques que j'ai déployées, tant bien que mal, pour faire ma place sur ce « terrain sensible» (Bouillon, Fresia, Tallio, 2005). Car, comme le signale S. Rullac (2005), mener une ethnographie au long cours au contact des SDF ne relève pas de l'évidence méthodologique et soulève un certain nombre de difficultés qu'il faut bien contourner ou accepter pour réaliser le projet. Quelques précédents notables attestent d'ailleurs de ces difficultés mais
2 Nancy s'articule à un centre-ville « à taille humaine » (entendons praticable à pied) et comprend moins de sans-abri que Paris, Marseille ou encore Lyon. Si bien qu'il y est possible d'observer et d'analyser un véritable réseau d'interconnaissance localisé. 
aussi, parallèlement, de tout l'intérêt qu'il peut y avoir à déployer l'approche ethnographique par rapport à cet objet de recherche (Snow, Anderson, 1993 ; Gaboriaux, 1993 ; Pimor, 2014 ; Bruneteaux, 2018).

\section{Stratégie de l'immersion ethnographique : lectures et anticipation de l'enquête}

Anticiper le déroulement de l'immersion ethnographique permet d'éviter certaines déconvenues, notamment au moment d'entrer sur le terrain. Car, avant d'être une méthode d'observation, l'ethnographie se caractérise comme une «méthode de relation », c'est-à-dire qu'elle est conditionnée par les relations qui se développent et s'entretiennent entre l'ethnographe et ses informateurs : la personne du chercheur étant alors partie prenante de son objet d'étude et décisive quant aux relations et effets d'enquête qui peuvent s'imposer sur le terrain (Mauger, 1991 ; Bizeul, 1998). Cette première partie relate la stratégie que j'ai mise en place pour entrer sur le terrain tandis que la seconde développe plutôt les tactiques qui ont été déployées en situation, au coup par coup, relativement à la stratégie énoncée (De Certeau, 1990).

Il a d'abord fallu réfléchir aux registres relationnels dans lesquels j'allais m'engager et qui seraient bénéfiques (ou contre-productifs, ou risqués) compte tenu des attendus de la recherche. Étant un homme blanc, d'une trentaine d'années, jeune chercheur issu d'un milieu ouvrier typique des cités minières de Lorraine, connaisseur de la ville et de ses marges, ayant moi-même « fait la route » en camion aménagé pendant plusieurs mois, j'étais a priori en mesure de m'adapter à ce monde social que je connaissais peu, de m'y sentir à mon aise et de développer des liens personnels et familiers (si ce n'est affectifs) avec des personnes dont les propos et les comportements populaires (Hoggart, 1970) (parfois déviants, du moins marginaux) ne m'étaient pas tout à fait étrangers. Certes, tant objectivement qu'aux yeux des enquêtés, je demeurais un petit-bourgeois intellectuel écrivant des livres et donnant des cours, ce qui relativise ma proximité avec le terrain et réinjecte de la distance sociale entre eux et moi. J'escomptais néanmoins «neutraliser» autant que possible la situation d'enquête, ou du moins maîtriser tant bien que mal les effets d'enquête liés à l'asymétrie indépassable de la relation enquêteur/enquêté rendant illusoire cette neutralisation complète : à cet égard, la négociation se joue notamment à travers les «manipulations plus ou moins conscientes de la définition de la situation d'enquête » (Mauger, 1991). Qui plus est, la distance sociale, même manifeste, n'exclut pas la proximité relationnelle, même si elle induit des effets sur la nature des relations (potentiellement instrumentalisées, dominées, fantasmées...).

Je comptais donc m'appuyer sur ce qui me rapprochait des individus que j'allais rencontrer, notamment en adaptant ma tenue, mon langage, mes comportements et mes propos afin d'apparaître comme un allié, un jeune curieux, un intellectuel marginal, plutôt que comme un chercheur universitaire représentant les classes et les normes dominantes. Quand bien même l'asymétrie structurelle de la relation d'enquête subsistait, c'était une posture adaptée aux objectifs que je m'étais fixés. Accéder à d'autres situations, d'autres moments, d'autres lieux et d'autres comportements que ce que connaissent les travailleurs sociaux supposait en effet que je m'intègre dans le monde de la rue en personne et, dans la mesure du possible, en tant qu'allié des sans-abri. Tout du moins, il me semblait que j'avais peu de chances d'obtenir des données intéressantes si j'étais assimilé, ou simplement associé, aux travailleurs sociaux - ou, pire encore, à la police. 
À l'été 2017, en amont du lancement du terrain, je décidai donc de cette nécessité indispensable de m'inscrire dans des relations personnalisées a priori détachées de tout enjeu institutionnel. C'est pour cette raison que j'ai abordé le terrain « par le bas », c'est-à-dire par la rue, en rencontrant et côtoyant les personnes d'abord dans l'espace public et dans la sphère quotidienne, avant de les suivre dans les services sociaux où je retrouvais des intervenants avec lesquels nous avions convenu que je sois traité anonymement. Mes relations avec les travailleurs sociaux se limitaient au minimum afin de pouvoir tenir cette position tandis que, à plusieurs reprises, j'ai dû garder des informations pour moi au lieu de les divulguer aux travailleurs sociaux. À l'inverse, j'ai parfois obtenu des informations sur les personnes à la rue d'après les dossiers ou propos des travailleurs sociaux. Je devais alors faire mine de ne pas être au courant de certaines choses dans mes relations avec les enquêtés (un passé toxicomane, une expérience carcérale, un épisode de violence accrue, etc.).

Ainsi, sans avoir été considéré comme «l'un des leurs » dans le monde de la rue - ce qui relève de l'illusion de l'enquêteur dissimulé - je tendais néanmoins à être identifié comme une personne indépendante et amicale, même si ce processus demandait à être régulièrement entretenu. Si un certain flou demeurait quant à mon identité, pour la majorité de mes informateurs il semblait clair, au fur et à mesure des preuves que j'apportais de mon engagement et de «l'abaissement des barrières » (Goffman, 1973a, p. 189), que j'étais de leur côté et qu'ils n'avaient pas à craindre ma présence.

Par ailleurs, un détour par la littérature scientifique permet d'identifier des éléments qui peuvent aider à la construction de la stratégie d'enquête. C'est par ce détour que j'ai notamment perçu toute la fragilité des données « déclaratives » lorsqu'elles ne sont pas confrontées à des observations d'ordre comportemental (Bizeul, 2007 ; Peneff, 2009) ou recueillies sur la durée et dans divers contextes (Mauger, 1991 ; Bruneteaux, Lanzarini, 1998 ; Bruneteaux, 2018). Ceci est d'autant plus important que les sans-abri sont amenés à multiplier les récits d'eux-mêmes (face aux travailleurs sociaux, aux bénévoles et aux passants qui interrogent toujours les trajectoires de vie) et donc à développer des stratégies de présentation de soi (Lanzarini, 2000), des « discours-écrans » et de l'onirisme social (Bruneteaux, 2016) relatifs à l'identité narrative de survie (Pichon, 2010). Les apports de travaux antérieurs invitent donc à aller voir l'envers du décor, c'est-à-dire ce qui se loge derrière les paroles, dans l'infra-quotidien des routines, intimités et activités difficilement conscientisées ou difficilement avouables ; ce qui est intéressant sur ce terrain comme ailleurs.

Il est aussi important de s'imprégner des logiques internes au monde qu'on se propose d'étudier et ainsi d'éviter certains impairs afin d'entrer plus facilement en relation avec les enquêtés. Par exemple, j'avais compris avant d'entrer sur le terrain que la manche pouvait être considérée comme un travail par certains sans-abri (Pichon, 2010 ; Saporiti, 2015) ou du moins que cette activité s'effectuait de manière organisée, quasi ritualisée, et constituait à ce titre un moment où je risquais d'être dérangeant. J'ai donc pris soin de ne pas déranger les personnes que j'abordais au moment de la manche, en leur demandant concrètement si je pouvais m'asseoir un peu et en ne m'y arrêtant d'abord que quelques minutes (il m'arrivait quand même d'être congédié : «Désolé, mais je dois faire mes pièces, si on discute c'est mort!»). Il en allait de même en ce qui concerne l'intrusion dans des lieux occupés (parkings souterrains, coins de rue, squats...), même lorsqu'il s'agit d'espaces publics (Céfaï, Gardella, 2011).

Je savais aussi que, dans le monde de la rue comme ailleurs, les relations (et la relation d'enquête plus particulièrement) peuvent être instrumentalisées par les uns et les autres, en quête de profits individuels, d'alliés ou de prises de paroles au nom du collectif (Mauger, 1991 ; Lanzarini, 2000). Si bien que 
je pris garde, dans les premiers mois de l'immersion, de ne pas apparaître comme un «client » potentiel, c'est-à-dire comme une personne qu'on peut « taxer » et qui est d'abord considérée au regard de ce qu'elle peut apporter (en termes de services ou en termes matériels). Certes, comme j'avais un logement et un travail - ce que mes enquêtés savaient -,j'étais régulièrement sollicité avant que ces demandes ne cessent devant mon impossibilité d'y répondre (je n'avais jamais plus de quelques euros sur moi) et devant l'identification plus personnelle qui s'est développée au fur et à mesure.

Enfin, entre représentations collectives, fantasmes et réalités, j'imaginais également, nourri par des lectures, que le monde de la rue (et la Zone plus précisément) n'était pas exempt de drogues, de violences et de risques auxquels j'allais probablement être confronté (Fernandez, 2010 ; Beauchez, 2017). Il fallait donc se préparer à ces risques physiques et psychologiques en amont de l'enquête, notamment en m'inspirant d'exemples ethnographiques dans des milieux a priori dangereux (Bourgois, 2013 ; Venkatesh, 2017). C'est dans ce cadre qu'un psychologue a été mis à ma disposition par l'association ARS tout au long de l'immersion ${ }^{3}$. J'ai également été accompagné par l'ARS pour m'informer sur les maladies, infections et contagions auxquelles je m'exposais en fréquentant les sans-abri et en partageant leurs activités. Je m'appliquais aussi à anticiper, sous forme de projection mentale, les moments où je risquais de me mettre en danger (en accompagnant des personnes peu connues dans des endroits clos, par exemple, ou en étant considéré comme une personne qui a de l'argent sur elle et, à ce titre, potentiellement intéressante à dépouiller).

Plus précisément - et rétrospectivement - on peut dire que l'immersion s'est déroulée en trois phases stratégiques, qui se chevauchent nécessairement en fonction de l'évolution des liens avec les uns et les autres. J'avais passé 8 mois sur le terrain et, à la faveur d'un mélange de bricolage et d'anticipation stratégique, il s'avère que je m'étais progressivement intégré dans le monde de la rue, d'abord par une phase de familiarisation, puis par une phase d'intégration et, enfin, à travers une phase de confrontation. Chacune de ses phases ayant donné lieu à l'émergence d'obstacles et de difficultés (des craintes concernant mon projet, des refus de coopérer, des mises à l'épreuve...) qui se levaient petit à petit ou qui se cristallisaient, freinant le développement de relations personnelles.

\section{Des techniques de négociation pour entrer et se maintenir sur le terrain}

Il me faut donc à présent détailler les tactiques ad hoc que j'ai mises en place en cohérence avec la stratégie méthodologique énoncée ci-dessus, bien que cette stratégie ait été le fruit de l'improvisation autant que de l'anticipation : les enjeux principaux consistent à assurer l'entrée sur le terrain, à s'y maintenir et à y trouver une place (ou des places) qui permette (nt) d'observer et de connaître les aspects de la réalité sociale visés par la recherche.

La question de l'entrée sur le terrain a été brièvement abordée par E. Goffman dans un texte intitulé On Fieldwork (1989). Des réflexions à ce sujet apparaissent également de manière plus éparse dans le recueil de traductions dirigé par D. Céfaï (2010) et dans le manuel méthodologique de S. Beaud et F. Weber (2010). Bien que ce soit là une question traditionnellement posée dans le cadre de la méthode ethnographique, elle fait parfois l'objet de nondits (Caratini, 2012). Toujours est-il que l'entrée sur le terrain - c'est-à-dire les premiers contacts et les prises de position qui se jouent au début de l'enquête - conditionne grandement l'immersion ethnographique et la fécondité des perspectives qui peuvent émerger ensuite. Pour ma part, relativement aux
3 Je remercie d'ailleurs CharlesHenry Lelimouzin pour son accompagnement psychologique et les échanges que nous avons pu avoir sur les personnes rencontrées dans la mesure où il s'est spécialisé dans l'intervention auprès de ces publics. Nos séances favorisaient une réflexivité indispensable, notamment en ce qui concerne l'évolution des relations enquêteur/ enquêté et les interprétations que je proposais sur le comportement de mes informateurs : ces échanges m'ont notamment aidé à objectiver les effets d'enquête ici relatés. 
objectifs fixés d'établir des relations personnalisées avec mes informateurs et de participer personnellement à leur vie quotidienne, plusieurs dimensions méritent d'être répertoriées.

\section{La présentation de soi et la définition de la situation}

La réalisation de l'enquête est d'abord conditionnée par la manière dont nous sommes identifiés par nos informateurs, si bien qu'il faut s'interroger sur les ressorts et effets de la présentation de soi (Girola, 1996) et de la définition de la situation (Mauger, 1991). «Qui est-on? ? et « Que fait-on ici ? » sont les deux questions centrales dont il convient de maîtriser les réponses, autant que faire se peut, notamment par la mise en scène interactionnelle. Dans le langage de Goffman (1973b), la présentation de soi relève d'un travail de figuration et de gestion des apparences qui aide à orienter la définition de la situation et le « cadre de l'expérience » (Goffman, 1991) : cela passe notamment par la tenue corporelle et langagière, par le fait d'être associé à des personnes ou des institutions et par la manière de présenter son projet : « Le savoir-faire de l'enquêteur réside pour une bonne part dans sa capacité de manipulation de la situation» (Mauger, 1991, p. 131).

C'est pour cela que j'ai choisi de mobiliser une certaine face (personnelle) de moi-même tout en laissant en retrait la face plus professionnelle. À savoir que j'ai adopté une tenue vestimentaire, corporelle et langagière propre à mes expériences de vie personnelles (marginalisées) pour faciliter la proximité relationnelle, sans pour autant neutraliser la distance sociale entre eux et moi. Si bien que je me suis confectionné une tenue de terrain destinée à être toujours la même : une paire de chaussures de skate, un jean large, une ceinture décorée de dessins tribaux, un sweat-shirt noir à capuche, un gros blouson foncé, un keffieh et un béret. Je portais systématiquement un sac en bandoulière contenant mon enregistreur, des fiches de notes, mes livres précédents, un peu de nourriture et - par sécurité (réelle ou fantasmée) - une bombe lacrymogène. Au-delà de la volonté d'afficher mon appartenance relative aux cultures de la rue, cet accoutrement n'affichait pas de richesse, favorisait mon identification répétée et m’offrait la possibilité de me salir sans scrupule. Cet habit me faisait aussi passer pour un Zonard aux yeux des bénévoles, des passants et de sans-abri que je n'avais pas encore rencontrés, notamment lorsque je faisais la manche ou que je fréquentais les services sociaux. Ce qui offrait l'avantage « d'apprendre par corps » (Faure, 2000), c'est-à-dire d'être véritablement traité comme un sans-abri à certains moments ${ }^{4}$ et d'incorporer certaines postures indigènes (traverser la rue entre les voitures, faire la manche assis par terre, être indifférent au regard des passants, mobiliser spontanément le jargon de la rue, etc.). Cette incorporation restait cependant partielle : un soir durant lequel je mangeais avec les sans-abri au service alimentaire, je me suis empressé de décroiser mes jambes après m'être aperçu du décalage qu'affichait cette posture (perçue comme féminine ou intellectuelle) avec le rôle que je présentais habituellement dans la rue. Je devais également rester attentif à ne pas prononcer de formules perçues comme intellectuelles telles que « en l'occurrence » ou « en dépit de... », ce qui m’échappait parfois et venait rappeler la distance sociale dans la relation ethnographique : d'ailleurs, mes interlocuteurs ne manquaient pas de se moquer de mes « phrases d'écrivain ».

J'ai raisonné de la même manière à propos de mon téléphone portable. Afin de n'attirer aucune convoitise, mais aussi pour des raisons pratiques, je me suis muni d'un téléphone ancienne génération, avec des touches et coulissant. Cela me permettait d'une part de réduire la distance sociale manifeste entre eux et moi, mais aussi et surtout de prendre des notes sur le portable tout en observant des situations, et ce, même sous la pluie (grâce aux touches
4 Ainsi d'une fois où j'ai été contrôlé violemment par la police, des nombreuses fois où, faisant la manche accompagné d'un «vrai » sans-abri, j'ai pu recevoir des insultes, des railleries ou des compassions humiliantes de la part de passants, ou encore de la bienveillance (parfois excessive et infantilisante) exprimée par les bénévoles dans les services alimentaires. 
sensibles des anciens téléphones). Mes premières prises de notes se faisaient d'ailleurs via cet outil, par l'envoi de pense-bêtes (par SMS envoyés à mon propre numéro) qui étaient ensuite repris et complétés dans le journal de bord. Mes enquêtés n'étaient cependant pas dupes et me le faisaient parfois savoir : «Enfait, tu prends des notes sur ton portable ou quoi? ? », ce qui pouvait se justifier compte tenu du projet que j'avais annoncé, cela ne rompait pas le « cadre primaire » construit (Goffman, 1991).

Gagner la confiance nécessite de savoir rester à sa place, de ne pas forcer les interactions (en posant « trop » de questions par exemple ${ }^{5}$ ) et de participer aux activités banales des enquêtés. Ainsi, j'adaptais également mon langage, mes propos et attitudes corporelles, sans rechigner à m'asseoir par terre ou à raconter des expériences personnelles qui me rapprochaient des leurs ou de leurs sujets de discussion (comme mes voyages en camion, quelques expériences sexuelles ou délictueuses, des soirées bien arrosées, etc.). Dans l'ensemble, je jouais de l'imitation en reprenant progressivement à mon compte leurs expressions et thématiques indigènes afin de sympathiser au sens propre ${ }^{6}$. Cela dit, je restais un intellectuel à qui l'on demande de corriger un CV ou de remplir un document administratif, et une personne insérée à qui on peut « taxer » un appel téléphonique, une paire de gants ou une bière...

Ensuite, afin de limiter les doutes sur les raisons de ma présence répétée, j'ai tâché, dans la mesure du possible, d'être introduit auprès de certains sans-abri par des connaissances qui avaient déjà leur confiance ${ }^{7}$. Je devais néanmoins m'assurer d'être présenté en personne et non comme représentant une institution. J'étais alors introduit par ce type de phrase : «Tiens, je suis venu te présenter Thibaut, il fait une recherche sur la rue, c'est un ami, j'espère que tu pourras l'aider... en tout cas tu peux avoir confiance en lui. » Il fallait alors tenir compte du fait que je serais ensuite identifié comme un allié de ces «passeurs », bénéficiant ainsi de leur renommée et de leurs alliés mais héritant parallèlement de leurs opposants éventuels (ainsi de Thomas ${ }^{8}, 34$ ans, qui avait été en conflit avec la bénévole de l'association Aides et qui refusait de me parler, du moins pendant les premiers mois de terrain).

$\mathrm{Au}$-delà des quelques individus ainsi rencontrés, durant la phase de familiarisation, je passais rituellement saluer les mendiants, m'asseyant quelques minutes à leur côté après leur avoir proposé une cigarette roulée, tout en leur expliquant mon projet lors des premières rencontres : «En fait, je vais traîner dans les rues pendant plusieurs mois, j'essaie d'écrire un bouquin sur la rue... on se reverra je pense. » Je joignais le geste à la parole en sortant de ma sacoche deux précédents ouvrages dont j'étais l'auteur ou le co-auteur. Cette mise en scène assurait le sérieux de mon travail (j'avais déjà écrit des livres, ce n'était pas du baratin!) et laissait peu de doutes sur les raisons de ma présence régulière ; elle imposait cependant d'emblée le rôle d'écrivain et la distance sociale structurelle qu'il inclut dans le rapport enquêteur/enquêté.

Parallèlement, je prenais soin de ne pas dire que je travaillais sur « les SDF » et préférais la formule plus neutre (et moins stigmatisante) d'un travail « sur la rue ». Dans un premier temps, je formulais mon projet en évoquant l'idée d'écrire un livre, sans parler de sociologie, d'enquête ou de recherche, un ensemble de vocables qui pouvait, à mon sens, prêter à confusion. C'est seulement durant la phase d'intégration que je précisais qu'il ne s'agissait pas d'un roman, mais d'un ouvrage de sociologie, sorte de « documentaire écrit » à travers lequel je cherchais à apporter une connaissance " de l'intérieur », basée sur leurs mots, leurs comportements, leur vie... critiquant au passage les discours médiatiques et politiques qui ont tendance à se situer en surplomb : [Moi] : «Ils parlent à votre place, sans savoir, sans avoir traîné avec vous... moi j'aimerais que ça soit vous les auteurs quasiment!»
5 Sur ce point, la postface de l'ouvrage classique de W. F. Whyte (2002, p. 332) est particulièrement éclairante.

6 Ainsi la prison devient « le shtar », la manche devient « la cheum », la drogue devient « la machine », tandis qu'au moment de se quitter, il n'est pas rare de se dire mutuellement «fais attention à toi » (ce qui suggère au passage l'incertitude inhérente au monde de la rue). Plus largement, je copiais les rites d'interaction qui ont cours dans la rue en effectuant des « checks » et des bises en guise de salutation.

7 Une ancienne toxicomane devenue travailleuse sociale m'a ainsi présenté deux de ses anciennes connaissances. Une bénévole de l'association Aides, elle-même partie prenante du monde de la rue depuis plus de dix ans, m'a introduit auprès de nombreuses personnes en effectuant des rondes dans la ville à mes côtés. Enfin, Amandine Turri-Hoelken, documentariste et anthropologue, m'a également fait bénéficier de sa connaissance de la Zone (elle avait produit, un an auparavant, un documentaire photographique avec les Zonards de Nancy que j'allais désormais fréquenter) et permis d'être identifié positivement par un certain nombre de futurs informateurs.

8 Précisons que les noms des informateurs cités sont des noms d'emprunt. 
Durant cette première phase, il n'était pas question de sortir mon enregistreur et d'effectuer des entretiens formels : le cadre et la définition des relations en construction ne s'y prêtaient pas du tout. Je ne voulais pas mettre en avant mon rôle de chercheur mais plutôt celui d'allié, de potentiel copain écrivant sur leur vie (comme certains sans-domicile qui écrivent également). Cette posture délicate - semi-transparente, semi-dissimulée - n'a pas toujours été facile à tenir et supposait une attention continue de ma part. Mais cette définition de la situation s'est globalement imposée au fur et à mesure que je faisais la preuve à la fois du rôle d'écrivain et du rôle d'allié-copain qui n'étaient pas fondamentalement incompatibles.

Cette approche allait de pair avec l'affirmation, dès les premiers contacts, du fait que je n'avais pas grand-chose à leur apporter et que je n'étais pas spécialement là pour les aider, mais au contraire, que j'avais besoin d'eux, qu'eux pouvaient m'aider à mieux comprendre leur monde. Je retournais ainsi le rapport de dépendance dans lequel ils sont régulièrement cantonnés (à la manche, dans les services sociaux, avec les bénévoles...) en les plaçant en position de dominer la relation par leur expertise, quand bien même j'étais en position dominante structurellement. Je rappelais ainsi que jeétais ignorant et que j'avais besoin d'eux. Ainsi, l'asymétrie structurelle inhérente à l'enquête en milieu populaire (Mauger, 1991) était ponctuellement contrebalancée par une sorte d'asymétrie inversée, localisée et située, relative à leur expérience du monde de la rue.

\section{L'entretien de la relation. Une tension entre proximité et distance sociale}

J'avais été surnommé « l'écrivain » et j'étais bien identifié par la majorité des sans-abri présents à Nancy cet hiver 2017-2018. Si bien qu'une fois entré sur le terrain à travers les tactiques favorisant l'acceptation de ma présence et conditionnant les futures relations, il me fallait encore entretenir les liens pour pouvoir accéder progressivement aux couches d'intimités et de vie privée qui pourraient se dévoiler ensuite ; du moins avec les personnes qui avaient accepté ma présence, d'une manière ou d'une autre. En effet, pour certains, comme Fred (35 ans) et Jeff (38 ans), j'étais devenu une sorte de porte-parole : ils témoignaient au nom des autres sans-domiciles de l'organisation et des aléas du monde de la rue. Pour d'autres, comme Popey (40 ans) et Vernon (41 ans), je faisais plutôt figure de confident : ces derniers se livraient sur leur biographie, leurs ressentis subjectifs et leurs difficultés personnelles. Pour d'autres encore, tels Clément (23 ans), Flavien ( 27 ans) ou Chris ( 27 ans), j'étais considéré comme un copain avec qui occuper son temps et partager de bons moments, sans qu'ils ne parlent spontanément de la rue ou de leur situation personnelle. Pour une part enfin, à l'instar de Cam (41 ans) et de Yannis (27 ans), je demeurais incompris dans ma démarche et source de méfiance : ils se tenaient à distance de moi - si ce n'est soupçonneux - en m'adressant la parole le moins possible, voire en quittant un groupe lorsque j'y arrivais.

Si j'étais désormais intime avec plusieurs Zonards qui m'invitaient à passer « chez-eux » (au parking, au foyer d'urgence, au camion...), je devais encore « briser la glace » avec d'autres sans-domicile (clochards, étrangers, opposants).J'étais progressivement en position de mettre les individus devant leurs contradictions éventuelles, ayant entendu ou observé des éléments dissonants par rapport aux déclarations de tel ou tel informateur : je tirais ainsi parti de l'insertion préalable sur le terrain (Soutrenon, 2005).

Finalement, force est de constater que la relation d'enquête prenait des formes différentes en fonction du rapport de force symbolique qui s'instaurait avec mes enquêtés (dans leur diversité). Je devais me résoudre à n'accéder qu'à ce que les enquêtés étaient en mesure de me livrer, qu'il s'agisse de compor- 
tements, de paroles ou de moments partagés : la mise en scène du chercheur pour orienter la définition de la situation est « concurrencée » par la mise en scène des enquêtés, eux-mêmes attestant de définitions variables de la situation et d'intérêts divers à être en relation avec l'enquêteur.

Si un degré de familiarité a pu s'instaurer avec la majorité de mes enquêtés, c'est en déployant un ensemble de tactiques destinées à gagner leur confiance et à maximiser mon accès aux aspects de la vie quotidienne les moins accessibles a priori. Cela passait notamment par l'expression de la réciprocité de la relation mais aussi par la maîtrise de mon engagement avec les uns et les autres : je devais ménager la chèvre et le chou, créer des alliances en limitant les oppositions qui pouvaient naître. Car les enquêtés ont également des attentes envers l'enquêteur : être bien vus et tirer avantage (matériel ou symbolique) de la relation d'enquête, être un personnage central du futur livre, être un « enquêté modèle » qui ne correspond pas aux aspects les plus stigmatisants du statut de sans-domicile, etc. Par conséquent, la réciprocité de la relation se jouait dans la compréhension de leurs intérêts et de leur positionnement vis-à-vis de l'enquêteur.

C'est notamment par un subtil usage des modalités de don et contre-don que j'ai pu manier la relation d'enquête, dans une certaine mesure (car eux aussi maniaient cette relation). Je trouvais ainsi une place dans le monde de la rue, certes incertaine et labile en fonction des enquêtés. L'important était de n'être pas réduit au rôle de chercheur venu prendre (des informations, des observations, des témoignages...) sans rien donner, ni au rôle de « client » venu pour donner (de l'argent, des services, de l'aide...) sans rien prendre. Une épaisseur personnelle venait s'intercaler entre ces deux pôles de la relation enquêteur/enquêté : c'est cette proximité qui ouvrait l'accès à « l'envers du décor » et au « texte caché », tout en restant surplombée et conditionnée par l'asymétrie structurelle de la relation d'enquête.

Si je m'étais bien gardé de donner de l'argent, prétextant que je ne sortais jamais avec plus de quelques euros, j'étais néanmoins perçu comme un pourvoyeur de tabac, ce qui me permettait au passage de pouvoir échanger en fumant avec eux. Je rendais service à l'occasion, en allant chercher des affaires égarées, en donnant une multiprise électrique, en transportant un ticket-restaurant d'une personne à l'autre ou bien simplement en gardant un chien à la manche, pendant que le propriétaire s'en allait faire un tour (généralement pour acheter une boisson au supermarché, pour une prise de drogue dans un endroit retranché ou pour aller voir une connaissance postée ailleurs à la manche). Il me fallait prendre garde de ne pas me poser en « magister » (Mauss, 2007) dans le cas où mes dons auraient été excessifs, les plaçant alors sous une dette symbolique (et matérielle) les obligeant à mon égard. Et il fallait parallèlement accepter les dons (une bière, un sandwich, une écharpe, une casquette...) qui m'étaient faits à l'occasion, quand bien même je n'en avais pas besoin : aux fondements essentiels de la réciprocité, recevoir est aussi important que donner et rendre (Caillé, 2007).

Faire preuve de mes bonnes dispositions à leur égard est parfois passé par le cautionnement d'activités illégales (ce qui me permettait en même temps de confirmer que je n'étais pas là pour «balancer » et même que je faisais partie de leur camp, face aux policiers ou aux travailleurs sociaux par exemple). Ainsi des soirs où j'ai fait rentrer quelques bouteilles d'alcool dans les foyers d'hébergement, n'étant pas fouillé par les surveillants de nuit qui étaient informés de mon étude. C'est le cas également lorsque je roulais les joints d'un informateur après qu'il se soit injecté de la cocaïne, lorsqu'on me donnait des Subutex que je pouvais alors redonner à ma guise à mes informateurs privilégiés ou encore lorsque j’allais retirer un colis alimentaire avec ma carte d'identité pour le donner à l'Indien ( 56 ans) qui avait perdu ses papiers. Des alliances se créaient ainsi et je pouvais m'attirer la confiance de quelques 
réticents. Je prenais soin aussi de ne pas froisser ceux que je n'aidais pas, en leur témoignant de l'attention à un autre moment, mais des oppositions se cristallisaient néanmoins. Si ces démarches m'ouvraient l'accès à certaines personnes, elles me fermaient également l'accès à d'autres. Sur le plan éthique, comme tout un chacun dans la vie ordinaire, je voyais se nouer des rapports affinitaires et s'entériner des rapports de défiance qu'il fallait bien accepter.

La réciprocité de la relation, qu'il ne faut pas confondre avec la symétrie illusoire, se jouait aussi à travers ce que je livrais de personnel. En effet, dans la mesure où j'essayais d'obtenir des informations sur leur vie privée (présente et passée), il me semblait que je devais moi-même leur raconter des épisodes de ma vie. Je me gardais de raconter des tranches de vie accentuant la distance sociale et privilégiais les expériences et anecdotes me rapprochant de leurs expériences et de l'identité que je présentais sur le terrain. C'est ainsi que j'ai souvent été le premier à me livrer en partageant des détails intimes comme une forme de don de moi-même : la parole peut aussi entrer dans l'économie du don (Besozzi, 2017). La réciprocité passait également par l'absence de jugement négatif sur les activités et comportements des uns et des autres (ce qui reste une approche classique en sociologie). Ici, il s'agissait pour moi d'affirmer à chaque occasion qui se présentait que je n'étais pas là pour juger, que je ne condamnais pas la toxicomanie, la délinquance, la débrouille, la violence... Au besoin, je racontais une anecdote personnelle attestant que je connaissais la violence, la drogue, la délinquance, etc. D’autres sujets étaient abordés en fonction de ma proximité avec les informateurs : je pouvais parler sérieusement de théorie avec Fred (35 ans) qui avait lu Marx et Lévi-Strauss, de sexe avec Julien (38 ans) qui arrivait parfois à se faire inviter chez des citadines qu'il avait séduites, ou encore de mon parcours professionnel avec Jeff (38 ans) qui faisait figure d'informateur privilégié.

Mais le meilleur opérateur de consolidation des relations reste le fait de partager des moments ordinaires de sociabilité. Tuer le temps ensemble, boire quelques bières, fumer des cigarettes, partager un colis alimentaire sur les marches d'une église, faire la manche avec eux, nourrir les discussions sur les bagarres, les voitures, la drogue, le sport, etc. ont été autant de moments où se banalisait ma présence et où s'affichaient ma coopération et mon appartenance (certes relative) à leur monde. Si je n'étais que de passage dans leur monde, j'y prenais désormais part en tant que personne : l'identité de chercheur-écrivain semblait passer au second plan pour la majorité d'entre eux.

Le plus difficile, durant la phase de confrontation, était de mesurer mon engagement avec les uns et les autres. C'est-à-dire que, ayant fréquenté la majorité des personnes régulièrement présentes dans la Zone et les services sociaux, je pouvais me trouver en porte-à-faux vis-à-vis de personnes qui ne s'appréciaient pas du tout (alors même que je côtoyais les deux « camps ») ou bien encore me voir reprocher de passer plus de temps avec untel qu'avec tel autre. Je rappelais alors ma position en spécifiant : "Je ne suis pas dans le game moi... je n'ai pas d'ennemis, je discute avec tout le monde parce que tout le monde a des choses à m'apprendre. » Il fallait s'empresser de préciser - je l'ai appris à mes dépens - que, de ce fait, je gardais les choses pour moi, que je n'allais pas répéter les histoires qui m’étaient racontées. Ainsi de ce jour du mois de mars 2018 (après 6 mois passés sur le terrain) où Christophe (50 ans) me soupçonna d'avoir indiqué à d'autres l'endroit où il dormait. Je lui répondis alors de manière assez virulente : «Non mais ! Est-ce que j’ai déjà balancé le moindre truc que tu m'as raconté? ! Est-ce que je t'ai déjà dit quelque chose sur les autres?! Non! Alors t'inquiète pas là-dessus, j’ai pas le choix moi, si je fais pas gaffe je suis mort!» Tenir sa langue est d'ailleurs une règle communément admise dans le monde de la rue où les commérages fusent sous une forme 
anonymisée qui permet paradoxalement de ne dénoncer personne. Au regard de ces remarques, il est évident que tous ne m'ont pas livré des informations avec la même transparence.

La question de la sortie du terrain pourrait elle aussi faire l'objet de tactiques destinées à la faciliter. Pour ma part, il a seulement été question, au fur et à mesure de l'immersion, de rappeler (ponctuellement) que j'étais « là » pendant 7-8 mois et que j'allais arrêter de traîner dans les rues à partir du mois d'avril (2018) pour me concentrer sur l'écriture. Je précisais néanmoins que ça ne m'empêcherait pas de revenir dire bonjour à l'occasion, ce qui fût bien le cas. Mais, à chaque fois, cela restaurait de la distance sociale et semblait provoquer de l'incompréhension : la tension entre la proximité relationnelle et la distance sociale refaisait son apparition...

\section{Conclusion : tensions éthiques relatives à l'immersion ethnographique}

Si j’ai tenté de mettre en évidence les apports généraux de la méthode ethnographique ainsi que les tactiques qui peuvent permettre de contrôler les apparences et de maîtriser les relations qui se développent sur le terrain, il reste à signaler la principale difficulté à laquelle je me suis confronté, qui est d'ordre éthique.

Certes, l'immersion telle que je l'ai pratiquée s'est avérée particulièrement féconde pour accéder à l'intimité, à la vie privée et aux activités cachées du monde de la rue. Il reste que cela s'est opéré au prix d'une tension mentale interne (et d'une tension relationnelle) qui reste vive aujourd'hui encore. Après tout, j'avais vraiment rusé pour avoir accès à mes informateurs, frôlant parfois le mensonge, la manipulation ou l'illégalité. Jusqu'à quel point la connaissance scientifique justifie-t-elle les tactiques ici décrites ? Il me semble que les limites éthiques que nous sommes prêts à atteindre dépendent fortement de notre personnalité, de ce que nous sommes aptes à endurer, mais aussi des objectifs fixés par la recherche. Dans mon cas, il m'est apparu compliqué, voire impossible, d'accéder (même dans une mesure restreinte) aux activités privées, à l'intimité et au texte caché des sans-abri sans recourir à un certain nombre de mises en scène pour entrer et me maintenir sur le terrain en personne, mais aussi pour pouvoir recouper les données observées, les propos recueillis en entretien et les discours énoncés en situation. Pour les besoins de l'enquête, j'ai dû taire certaines réticences (face à l'héroïne, à la violence, à la délinquance...) et me comporter parfois en désaccord avec mes principes personnels.

Je me préservais du sentiment de duplicité en m’appliquant à reconnaître et à valoriser sincèrement les personnes que j'ai rencontrées, tout en m'imaginant que, même s'ils ne savaient pas tout des raisons de ma présence et de mes liens professionnels avec les travailleurs sociaux, je me devais néanmoins d'être une sorte de porte-parole de leur réalité devant les instances décisionnaires de l'action sociale locale, quand il ne s'agissait pas de leur venir en aide plus ou moins directement. Comme le rappelle S. Chauvin (2017), il est inévitable que le chercheur ait ses « placards » sur le terrain, ses objectifs propres, ses formules implicites et ses non-dits. Dès lors, autant qu'il s'en saisisse pour maittriser sa recherche tout en préservant ses informateurs dans ses comptes rendus et dans les interactions (Fassin, 2015, p. 46-47). Pour ma part, que cela soit en réunion ou dans mes écrits, je m'appliquais à ne fournir aucune information qui aurait pu se retourner contre mes informateurs (le nombre et l'emplacement des squats, les activités illégales, les propos les plus 
critiques, etc.), notamment en rendant impersonnelles les déclarations sur lesquelles je m’appuyais et sans indiquer les endroits où se déroulaient les situations décrites.

On retiendra enfin qu'il ne faut pas s'illusionner sur la dissimulation de l'immersion et la proximité relationnelle : les mises en scène du chercheur trouvent leur parallèle dans la mise en scène des enquêtés face au chercheur. Comme le signale G. Mauger (1991), ces effets d'enquête doivent être utilisés comme du matériel d'analyse à part entière - donnant accès au système de valeur des enquêtés - plutôt que comme des obstacles à lever pour développer une hypothétique relation authentique. 


\section{Bibliographie}

BEAUCHEz Jérôme, 2017, « Zone stupéfiante : l'espace du deal », Espaces et sociétés, $\mathrm{n}^{\circ} 171, \mathrm{p} .55-72$.

Besozzi Thibaut, 2017, « Ce que le don dit et ce que le dire donne. Enquête sur la sociabilité des habitués d'un centre commercial », Revue du MAUSS, n ${ }^{\circ}$ 50, p. 211-226.

BEsozzi Thibaut, 2019, Le monde de la rue à Nancy: de l'errance chronique à la stabilisation?, Rapport de recherche, Université de Lorraine, 2L2S, $200 \mathrm{p}$.

Bizeul Daniel, 1998, « Le récit des conditions d'enquête : exploiter l'information en connaissance de cause », Revue française de sociologie, $\mathrm{n}^{\circ} 39$, p. 751-787.

Bizeul Daniel, 2007, «Que faire des expériences d'enquête? Apports et fragilité de l'observation directe $»$, Revue française de science politique, vol. 57, $\mathrm{n}^{\mathrm{o}} 1, \mathrm{p} .69-89$.

Boulllon Florence, Fresia Marion et Tallio Virginie (eds.), 2005, Terrains sensibles. Expériences actuelles de l'anthropologie, Paris, CEA-EHESS, $208 \mathrm{p}$.

BourgoIs Philippe, 2013, En quête de respect. Le crack à New York, $2^{\square}$ éd., Paris, Éditions du Seuil, 482 p.

BRUneteaux Patrick et LANZARINI Corinne, 1998, « Les entretiens informels », Sociétés contemporaines, $\mathrm{n}^{\circ}$ 30, p. 157-180.

Bruneteaux Patrick, 2016, Les mondes rêvés de Georges, Rennes, Presses Universitaires de Rennes, 335 p.

BRUneteaux Patrick, 2018, « Ethnographie et lien social. Pratique et éthique de la recherche auprès des résidents des foyers d'urgence », Bulletin de Méthodologie Sociologique, $\mathrm{n}^{\circ}$ 41, p. 39-89.

CAIllé Alain, 2007, Anthropologie du don, $2^{\square}$ éd., Paris, La Découverte, $280 \mathrm{p}$.

CARATINI Sophie, 2012, Les non-dits de l'anthropologie, Vincennes, Thierry Marchaisse, $192 \mathrm{p}$.

CÉfAÏ Daniel (eds.), 2010, L'engagement ethnographique, Paris, Éditions EHESS, $640 \mathrm{p}$.

CÉfAÏ Daniel et GARDELla Édouard, 2011, L'urgence sociale en action, Paris, La Découverte, $750 \mathrm{p}$.

CÉfAÏ Daniel, 2015, « Mondes sociaux. Enquête sur un héritage de l'écologie humaine à Chicago », SociologieS [En ligne], mis en ligne le 23 février 2015, consulté le 24 août 2020. URL : http://journals.openedition.org/ sociologies/4921.

Certeau De Michel, 1990, L'invention du quotidien, tome I : Arts de faire, $2^{\text {घ }}$ éd., Paris, Gallimard, 347 p.

Chauvin Sébastien, 2017, « Les placards de l'ethnographe », dans P. Leroux et E. Neveu (eds.), En immersion. Approches ethnographiques en journalisme, littérature et sciences sociales, Rennes, Presses Universitaires de Rennes, p. 163-174.

CHOPPIN Katia et GARDELLA Édouard (dir.), 2013, Les sciences sociales et le sans-abrisme. Recension bibliographique de langue française, SaintÉtienne, Publications de l'Université de Saint-Étienne, 348 p.

DAMON Julien, 2012, La question SDF. Critique d'une action publique, $2^{\circledR}$ éd., Paris, PUF, 408 p.

DAMON Julien, 2017, Exclusion : vers zéro SDF?, Paris, La documentation Française, 177 p.

FAssin Didier, 2015, La force de l'ordre. Une anthropologie de la police des quartiers, $2^{\unrhd}$ éd., Paris, Éditions du Seuil, 441 p. 
FAURE Sylvia, 2000, Apprendre par corps. Socio-anthropologie des techniques de danse, Paris, La Dispute, 279 p.

FERNANDEZ Jérôme, 2010, Emprises. Drogues, errance, prison : figures d'une expérience totale, Bruxelles, Larcier, $384 \mathrm{p}$.

GABORIAU Patrick, 1993, Clochard. L'univers d'un groupe de sans-abri parisiens, Paris, Julliard, $235 \mathrm{p}$.

GiRola Claudia, 1996, « Rencontrer des personnes sans abri. Une anthropologie réflexive », Politix, $\mathrm{n}^{\circ}$ 34, p. 87-98.

GoFFMAN Erving, 1973a, La mise en scène de la vie quotidienne, Tome 1: la présentation de soi, Paris, Éditions de Minuit, 256 p.

GoFFMAN Erving, 1973b, La mise en scène de la vie quotidienne, Tome 2 : les relations en public, Paris, Éditions de Minuit, 376 p.

Goffman Erving, 1974, Les rites d'interaction, Paris, Éditions de Minuit, $236 \mathrm{p}$.

GoFFMAN Erving, 1989, « On Fieldwork », Journal of Contemporary Ethnography, vol. 18, p. 123-132.

Goffman Erving, 1991, Les cadres de l'expérience, Paris, Éditions de Minuit, $573 \mathrm{p}$.

Hoggart Richard, 1970, La culture du pauvre, Paris, Éditions de Minuit, $424 \mathrm{p}$.

JosePh Isaac, 2007, L'athlète moral et l'enquêteur modeste, Paris, Economica, $494 \mathrm{p}$.

LANZARINI Corinne, 2000, Survivre dans le monde sous-prolétaire, Paris, PUF, $296 \mathrm{p}$.

Leroux Pierre et Neveu Erik (eds.), 2017, En immersion. Pratiques intensives du terrain en journalisme, littérature et sciences sociales, Rennes, Presses Universitaires de Rennes, 428 p.

MAUger Gérard, 1991, «Enquêter en milieu populaire », Genèses, nº 6, p. 125-143.

Mauss Marcel, 2007, Essai sur le don, Paris, PUF, 248 p.

Peneff Jean, 2009, Le goût de l'observation. Comprendre et pratiquer l'observation participante en sciences sociales, Paris, La Découverte, $256 \mathrm{p}$.

Pichon Pascale, 2010, Vivre dans la rue. Sociologie des sans domicile fixe, Saint-Étienne, Publications de l'Université de Saint-Étienne, 228 p.

PIMOR Tristana, 2014, Zonards. Une famille de rue, Paris, PUF, 280 p.

REvUE Espaces et sociétés, 2017, $\mathrm{n}^{\circ}$ 171, «Zone : l'espace d'une vie en marge », Paris, Érès, 184 p.

RulLAC Stéphane, 2005, Et si les SDF n'étaient pas des exclus ? Essai ethnologique pour une définition positive, Paris, L'Harmattan, 148 p.

SAPORITI Lionel, 2015, Comprendre des vies de plus de dix ans dans la rue par une approche biographique menée dans la durée, thèse de doctorat de sociologie, Université de Strasbourg, 438 p.

Scотт James C., 2008, La domination et les arts de la résistance. Fragments du discours subalterne, Paris, Éditions Amsterdam, 272 p.

SNow David et ANDERSon Leon, 1993, Down on their Luck : A Study of Homeless Street People, Oakland, University of California Press, 406 p.

SoUtRENON Emmanuel, 2005, « Le "questionnaire ethnographique". Réflexion sur une pratique de terrain », Genèses, nº 60, p. 121-137.

Venkatesh Sudir, 2017, Gang Leader for a Day, $2^{\circledR}$ éd., New York, Penguin Press, 355 p.

Whyte William F., 2002, Street Corner Society, $2^{\natural}$ éd., Paris, La Découverte, $403 \mathrm{p}$. 